\title{
A New RH-Regular Matrix Derived by Jordan's Function and Its Domains on Some Double Sequence Spaces
}

\author{
Sezer Erdem ${ }^{1}$ and Serkan Demiriz (iD $^{2}$ \\ ${ }^{1}$ Battalgazi Farabi Anatolian Imam Hatip High School, 44210 Malatya, Turkey \\ ${ }^{2}$ Department of Mathematics, Tokat Gaziosmanpasa University, 60250 Tokat, Turkey \\ Correspondence should be addressed to Serkan Demiriz; serkandemiriz@gmail.com \\ Received 8 February 2021; Revised 26 March 2021; Accepted 5 April 2021; Published 19 May 2021 \\ Academic Editor: Gen Qi Xu \\ Copyright (c) 2021 Sezer Erdem and Serkan Demiriz. This is an open access article distributed under the Creative Commons \\ Attribution License, which permits unrestricted use, distribution, and reproduction in any medium, provided the original work \\ is properly cited. \\ In the present study, we introduce a new RH-regular 4D (4-dimensional) matrix derived by Jordan's function and define double \\ sequence spaces by using domains of $4 \mathrm{D}$ Jordan totient matrix $\mathfrak{F}^{t}$ on some classical double sequence spaces. Also, the $\alpha$-, $\beta(\vartheta)$-, \\ and $\gamma$-duals of these spaces are determined. Finally, some classes of 4D matrices on these spaces are characterized.
}

\section{Introduction and Preliminaries}

The Jordans's function $J_{t}: \mathbb{N} \longrightarrow \mathbb{N}, k \longmapsto J_{t}(k)$ is described as the number of $t$-tuples of positive integers all less than or equal to $k$ that form a coprime with $(t+1)$-tuples together with $k$, where $k, t \in \mathbb{N}$ and $\mathbb{N}=\{1,2, \cdots\}$. $J_{t}$ is entitled as Jordan's function after Camille Jordan, and it is one of many generalizations of the famous Euler-totient function $\varphi$. Since the equality $J_{t}\left(k_{1} k_{2}\right)=J_{t}\left(k_{1}\right) J_{t}\left(k_{2}\right)$ holds for the coprime numbers $k_{1}, k_{2} \in \mathbb{N}$, $J_{t}$ is multiplicative, and furthermore, it is an arithmetic function. If $a_{1}{ }^{b_{1}} a_{2}{ }^{b_{2}} a_{3} b_{3} \cdots a_{i}^{b_{i}}$ is the prime factorization of $k \in \mathbb{N}$ for $k>1$, in that case,

$$
J_{t}(k)=k^{t}\left(1-\frac{1}{a_{1}^{t}}\right)\left(1-\frac{1}{a_{2}^{t}}\right)\left(1-\frac{1}{a_{3}^{t}}\right) \cdots\left(1-\frac{1}{a_{i}^{t}}\right) .
$$

It is also significant to say that the Euler-totient function $\varphi$ is the special case of the Jordan's function $J_{t}$ for $t=1$. Other three important features of Jordan's function are

$$
\begin{aligned}
& \sum_{k \mid m} J_{t}(k)=m^{t}, \\
& \sum_{k \mid m} \frac{\mu(k)}{k^{t}}=\frac{J_{t}(m)}{m^{t}},
\end{aligned}
$$

and

$$
\sum_{k \mid m} \mu\left(\frac{m}{k}\right) k^{t}=J_{t}(m)
$$

where $\mu$ denotes the Möbius function (see [1]). Given any $k \in \mathbb{N}$, the function $\mu$ is described as

$$
\mu(k):= \begin{cases}1 & k=1, \\ (-1)^{i}, & k=a_{1} a_{2} \cdots a_{i}, \text { where } a_{1}, a_{2}, \cdots, a_{i} \text { are different prime numbers, } \\ 0, & a^{2} \mid k \text { for at least one prime number } a .\end{cases}
$$


If $a_{1}{ }_{1}^{b_{1}} a_{2}{ }^{b_{2}} a_{3} b_{3} \cdots a_{i} b_{i}$ is the prime factorization of $k \in \mathbb{N}$ such that $k>1$, in this fact, $\sum_{k \mid m} k \mu(k)=\left(1-a_{1}\right)\left(1-a_{2}\right)$ $\left(1-a_{3}\right) \cdots\left(1-a_{i}\right)$. For $m \neq 1$, the equality $\sum_{k \mid m} \mu(k)=0$ holds and $\mu\left(k_{1} k_{2}\right)=\mu\left(k_{1}\right) \mu\left(k_{2}\right)$, where $k_{1}, k_{2} \in \mathbb{N}$ are coprime.

A double sequence is a function such that $f: \mathbb{N} \times \mathbb{N}$ $\longrightarrow \zeta,(k, l) \longmapsto f(k, l)=x_{k l}$, where $\zeta$ denotes any nonempty set. Any linear subspace of the linear space $\Omega:=\left\{x=\left(x_{k l}\right)\right.$ : $\left.x_{k l} \in \mathbb{C}, k, l \in \mathbb{N}\right\}$ is named as double sequence space. Here, the symbol $\mathbb{C}$ represents the complex field. $x=\left(x_{k l}\right) \in \Omega$ is convergent in the Pringsheim's sense provided that for all $\xi>0$, there subsists $n_{\xi} \in \mathbb{N}$ with $\left|x_{k l}-\kappa\right|<\xi$ for $k, l>n_{\xi}$. In that case, $\kappa \in \mathbb{C}$ is named as the Pringsheim limit of $x$ and stated with $p-\lim _{k, l \rightarrow \infty} x_{k l}=\kappa . \mathscr{C}_{p}$ represents the space of all such $x$ which are called shortly as p-convergent. The space of all bounded double sequences is denoted by $\mathscr{M}_{u}$. Of particular interest is unlike single sequences, when $p-$ $\lim _{k, l \longrightarrow \infty} x_{k l}=\kappa$, it might be $x \notin \mathscr{M}_{u}$. The bounded sequences which are also $p$-convergent are indicated by $\mathscr{C}_{b p}$, that is, $\mathscr{C}_{b p}=\mathscr{C}_{p} \cap \mathscr{M}_{u} \cdot x=\left(x_{k l}\right) \in \mathscr{C}_{p}$ is entitled as regularly convergent if the limits $x_{k}:=\lim _{l} x_{k l},(k \in \mathbb{N})$ and $x_{l}:=\lim _{k} x_{k l}$, $(l \in \mathbb{N})$ subsist, and the limits $\lim _{k} \lim _{l} x_{k l}$ and $\lim _{l} \lim _{k} x_{k l}$ subsist and are equivalent to the $p-\lim x$. The set of all regularly convergent double sequences is represented by $\mathscr{C}_{r}$, and also, $\mathscr{M}_{u}, \mathscr{C}_{b p}$, and $\mathscr{C}_{r}$ are Banach spaces with the norm $\|x\|_{\infty}=\sup _{k, l \in \mathbb{N}}\left|x_{k l}\right|$. Moreover, $\mathscr{C} \mathcal{S}_{\vartheta}$ and $\mathscr{B} \mathcal{S}$ symbolize the spaces of all 9 -convergent and bounded series, respectively. It should be noted that the double sequence space $\mathscr{L}_{u}$ which was defined by Zeltser [2] is the special case of the space $\mathscr{L}_{s}$ described by

$$
\mathscr{L}_{s}:=\left\{\left(x_{k l}\right) \in \Omega: \sum_{k, l=1}^{\infty, \infty}\left|x_{k l}\right|^{s}<\infty\right\},(0<s<\infty),
$$

for $s=1$. It is also significant to advert that the space $\mathscr{L}_{s}$ is Banach space with

$$
\|x\|_{\mathscr{L}_{s}}=\left(\sum_{k, l}\left|x_{k l}\right|^{s}\right)^{1 / s} \cdot(1 \leq \mathcal{S}<\infty) .
$$

The concept of almost convergence which is more general than the convergence of single sequences was acquainted by Lorentz [3], and later, present idea was conveyed and examined for double sequences in [4]. The spaces of all almost convergent and almost null double sequences denoted by $\mathscr{C}_{f}$ and $\mathscr{C}_{f_{0}}$, respectively.

Consider that $x \in \Omega$ and describe $Y=\left(v_{m n}\right)$ as $v_{m n}:=$ $\sum_{k=1}^{m} \sum_{l=1}^{n} x_{k l},(m, n \in \mathbb{N})$. In that case, the pair $\left(\left(x_{m n}\right),\left(v_{m n}\right)\right)$ is entitled as double series. Here, the sequence $Y=\left(v_{m n}\right)$ is the sequence of partial sums of the double series.

Throughout this article, it will be used $\sum_{k, l}$ instead of $\sum_{k=1}^{\infty} \sum_{l=1}^{\infty}$, 4D instead of 4-dimensional, $\vartheta \in\{p, b p, r\},(1 / s)$ $+\left(1 / s^{\prime}\right)=1(1<s<\infty)$, and $m, n, k, l \in \mathbb{N}$. Double sequences $e^{m n}=\left(e_{k l}^{m n}\right)$, and $e$ described by

$$
e_{k l}^{m n}:= \begin{cases}1, & \text { if }(m, n)=(k, l), \\ 0 & \text { for the other cases }\end{cases}
$$

and $e=\sum_{m, n} e^{m, n}$. If $b_{m n k l}=0$ for $k>m$ or $l>n$ or both, $B=\left(b_{m n k l}\right)$ is named as triangular matrix, and also, if $b_{m n m n} \neq 0$, in that case, $B$ is called as triangle.

Let us consider $\Psi, \Lambda \subset \Omega$ and the $4 \mathrm{D}$ complex infinite matrix $B=\left(b_{m n k l}\right)$. If for all $x=\left(x_{k l}\right) \in \Psi,(B x)_{m n}=\vartheta-\sum_{k, l}$ $b_{m n k l} x_{k l}$ subsists and is in $\Lambda$ for each $m, n \in \mathbb{N}$, in that case, $B$ describes a matrix transformation from $\Psi$ into $\Lambda$, and it is written as $B: \Psi \rightarrow \Lambda$. $(\Psi: \Lambda)$ stands for the class of all $4 \mathrm{D}$ complex infinite matrices from $\Psi$ into $\Lambda$. In that case, $B \in(\Psi: \Lambda)$ if and only if $B_{m n} \in \Psi^{\beta(9)}$ and $B x \in \Lambda$ for all $x \in \Psi$, where $B_{m n}=\left(b_{m n k l}\right)_{k, l \in \mathbb{N}}$ and $m, n \in \mathbb{N}$.

The domain $\Psi_{B}^{(9)}$ of a $4 \mathrm{D}$ complex infinite matrix $B$ in a double sequence space $\Psi$ consists of the sequences whose $B$-transforms are in $\Psi$; that is,

$\Psi_{B}^{(9)}:=\left\{x=\left(x_{k l}\right) \in \Omega: B x:=\left(\vartheta-\sum_{k l} b_{m n k l} x_{k l}\right)_{m, n \in \mathbb{N}}\right.$ subsists and is in $\left.\Psi\right\}$.

The 2D Jordan matrix and its domain on the space of $\ell_{p}$ of absolutely $p$-summable single sequences are described and examined by Ilkhan et al. [5]. The $4 \mathrm{D}$ Euler-totient matrix $\Phi^{\star}$ and domains of this matrix on double sequences $\mathscr{L}_{s}, \mathscr{M}_{u}, \mathscr{C}_{p}, \mathscr{C}_{b p}$, and $\mathscr{C}_{r}$ were described and examined by Demiriz and Erdem [6] and Erdem and Demiriz [7].

Suppose that $R^{q t}, \mathrm{E}(\mathrm{r}, \mathrm{s}), \mathrm{B}(\mathrm{r}, \mathrm{s}, \mathrm{t}, \mathrm{u}), \tilde{B}, \Phi^{\star}$, and $B^{(r, s)}$ denote the 4D Riesz, Euler, generalized difference, sequential band, Euler-totient, and binomial matrices, respectively. The domains $\Psi_{B}$ of some 4 D triangle matrices $B$ in a space $\Psi$ are listed in Table 1.

\section{The 4D Jordan Totient Matrix and Some Double Sequences}

In this section, we define 4D Jordan totient matrix $\mathfrak{F}^{t}$ derived by Jordan's function, and we show that this matrix is $\mathrm{RH}$ regular. By using its domains on the spaces $\mathscr{M}_{u}, \mathscr{C}_{p}, \mathscr{C}_{b p}$, $\mathscr{C}_{r}$, and $\mathscr{L}_{s}$ for $0<s<\infty$, we introduce the spaces $\mathfrak{J}_{\infty}^{t}, \mathfrak{J}_{p}^{t}$, $\mathfrak{J}_{b p}^{t}, \mathfrak{J}_{r}^{t}$, and $\mathfrak{J}_{s}^{t}$ and also give inclusion relations related these newly described spaces. The reader can refer to the papers [15-30] and recent monograph [31] concerning with the domain of $4 \mathrm{D}$ triangles in the classical spaces of double sequences and related topics. 
TABLe 1: Some 4D triangle matrices and their domains.

\begin{tabular}{lccc}
\hline$B$ & $\Psi$ & $\Psi_{B}$ & Refer to: \\
\hline$R^{q t}$ & $\mathscr{L}_{s}$ & $R^{q t}\left(\mathscr{L}_{s}\right)$ & {$[8]$} \\
$E(r, s)$ & $\mathscr{L}_{p}, \mathscr{M}_{u}$ & $\varepsilon_{p}^{r, s}, \varepsilon_{\infty}^{r, s}$ & {$[9]$} \\
$B(r, s, t, u)$ & $\mathscr{M}_{u}, \mathscr{C}_{b p}, \mathscr{C}_{p}, \mathscr{C}_{r}, \mathscr{L}_{q}$ & $B\left(\mathscr{M}_{u}\right), B\left(\mathscr{C}_{b p}\right), B\left(\mathscr{C}_{p}\right), B\left(\mathscr{C}_{r}\right), B\left(\mathscr{L}_{q}\right)$ & {$[10]$} \\
$B(r, s, t, u)$ & $\mathscr{C}_{f}, \mathscr{C}_{f_{0}}$ & $B\left(\mathscr{C}_{f}\right), B\left(\mathscr{C}_{f_{0}}\right)$ & {$[11]$} \\
$\tilde{B}$ & $\mathscr{M}_{u}, \mathscr{C}_{b p}, \mathscr{C}_{p}, \mathscr{C}_{r}, \mathscr{L}_{q}$ & $\tilde{B}\left(\mathscr{M}_{u}\right), \tilde{B}\left(\mathscr{C}_{b p}\right), \tilde{B}\left(\mathscr{C}_{p}\right), \tilde{B}\left(\mathscr{C}_{r}\right), \tilde{B}\left(\mathscr{L}_{q}\right)$ & {$[12]$} \\
$\Phi^{\star}$ & $\mathscr{L}_{p}$ & $\Phi^{\star}\left(\mathscr{L}_{p}\right)$ & {$[6]$} \\
$\Phi^{\star}$ & $\mathscr{M}_{u}, \mathscr{C}_{b p}, \mathscr{C}_{p}, \mathscr{C}_{r}$ & $\Phi^{\star}\left(\mathscr{M}_{u}\right), \Phi^{\star}\left(\mathscr{C}_{b p}\right), \Phi^{\star}\left(\mathscr{C}_{p}\right), \Phi^{\star}\left(\mathscr{C}_{r}\right)$ & {$[7]$} \\
$B^{(r, s)}$ & $\mathscr{M}_{u}, \mathscr{C}_{p}, \mathscr{C}_{b p}, \mathscr{C}_{r}$ & $\mathscr{B}_{\infty}^{r, s}, \mathscr{B}^{r, s}, \mathscr{B}_{b p}^{r, s}, \mathscr{B}_{r e g}^{r, s}$ & {$[13]$} \\
$B^{(r, s)}$ & $\mathscr{C}_{f}, \mathscr{C}_{f_{0}}$ & $\mathscr{B}_{f}^{r, s}, \mathscr{B}_{f_{0}}^{r, s}$ & {$[14]$} \\
\hline
\end{tabular}

Now, we describe the $4 \mathrm{D}$ Jordan totient matrix $\mathfrak{J}^{t}=$ $\left(j_{m n k l}^{t}\right)$ as follows:

$$
j_{m n k l}^{t}:= \begin{cases}\frac{J_{t}(k) J_{t}(l)}{(m n)^{t}} & k|m, l| n, \\ 0, & \text { for the other cases, }\end{cases}
$$

for each $t \in \mathbb{N}$. It should be noted that the 4D Euler-totient matrix $\Phi^{\star}$ defined by Demiriz and Erdem [6] is the special case of the matrix $\mathfrak{J}^{t}$ for $t=1$. Moreover, it is clear that $\mathfrak{J}^{t}$ is a triangle, and the $\mathfrak{J}^{t}$-transform of any $x=\left(x_{k l}\right) \in$ $\Omega$ is described as

$$
y_{m n}:=\left(\mathfrak{J}^{t} x\right)_{m n}=\frac{1}{(m n)^{t}} \sum_{k|m, l| n} J_{t}(k) J_{t}(l) x_{k l}
$$

The inverse $\left(\mathfrak{J}^{t}\right)^{-1}=\left(j_{m n k l}^{t^{-1}}\right)$ of the triangle matrix $\mathfrak{J}^{t}$ is calculated as

$$
j_{m n k l}^{t-1}:= \begin{cases}\frac{\mu(m / k) \mu(n / l)}{J_{t}(m) J_{t}(n)}(k l)^{t}, & k|m, l| n, \\ 0, & \text { for the other cases. }\end{cases}
$$

In that case, $x_{m n}$ is obtained by applying $\left(\mathfrak{\Im}^{t}\right)^{-1}$ to $(10)$ as

$$
x_{m n}=\sum_{k|m, l| n} \frac{\mu(m / k) \mu(n / l)}{J_{t}(m) J_{t}(n)}(k l)^{t} y_{k l} .
$$

Definition 1 (see $[32,33]$ ). For every $x \in \mathscr{C}_{b p}$, if $B x \in \mathscr{C}_{p}$ and $b p-\lim x=p-\lim B x$, then $B$ is called as RH-regular.
Lemma 2 (see $[32,33])$. A $4 D$ matrix $B=\left(b_{m n k l}\right)$ is $R H$ regular if and only if

$$
\begin{aligned}
& R H_{1}: p-\lim _{m, n \longrightarrow \infty} b_{m n k l}=0(k, l \in \mathbb{N}), \\
& R H_{2}: p-\lim _{m, n \longrightarrow \infty} \sum_{k, l} b_{m n k l}=1, \\
& R H_{3}: p-\lim _{m, n \longrightarrow \infty} \sum_{k}\left|b_{m n k l}\right|=0(l \in \mathbb{N}), \\
& R H_{4}: p-\lim _{m, n \longrightarrow \infty} \sum_{l}\left|b_{m n k l}\right|=0(k \in \mathbb{N}), \\
& R H_{5}: \sum_{k, l}\left|b_{m n k l}\right| \text { is } p-\text { convergent, } \\
& R H_{6}: \sum_{k, l>L}\left|b_{m n k l}\right|<K \text { for at least } K, L \in \mathbb{N} .
\end{aligned}
$$

Theorem 3. The $4 D$ Jordan totient matrix $\mathfrak{\Im}^{t}$ described by (9) is RH-regular.

Proof. Since $\left(J_{t}(k) / m^{t}\right) \longrightarrow 0(m \longrightarrow \infty)$ and $\left(J_{t}(l) / n^{t}\right) \longrightarrow$ $0(n \longrightarrow \infty)$ from the regularity of the $2 \mathrm{D}$ Jordan totient matrix [5], we obtain that $\left(J_{t}(k) J_{t}(l) /(m n)^{t}\right) \longrightarrow 0(m$, $n \longrightarrow \infty)$. Thus, RH-1 holds. From the equality

$$
\sum_{k, l} \frac{J_{t}(k) J_{t}(l)}{(m n)^{t}}=\sum_{k|m, l| n} \frac{J_{t}(k) J_{t}(l)}{(m n)^{t}}=1,
$$

we see that RH-2 holds. Since

$$
\sum_{k}\left|j_{m n k l}^{t}\right|=\sum_{k} \frac{J_{t}(k) J_{t}(l)}{(m n)^{t}}=\frac{J_{t}(l)}{n^{t}} \sum_{k \mid m} \frac{J_{t}(k)}{m^{t}}=\frac{J_{t}(l)}{n^{t}},
$$

then $p-\lim _{m, n \longrightarrow \infty} \sum_{k}\left|j_{m n k l}^{t}\right|=0$ for each $l$ and RH-3 holds. It can be shown similar with RH-3 that the $4 \mathrm{D}$ Jordan totient matrix satisfies RH-4. From the relation $\sum_{k, l}\left|j_{m n k l}^{t}\right|=1$, we see that RH-5 holds. By using the relation $\sum_{k, l} j_{m n k l}^{t}=1$ and 
the positivity of the matrix $\mathfrak{J}^{t}$, it is clear that the condition $\mathrm{RH}_{6}$ satisfies.

Now, we introduce the double sequence spaces by using the domains of $4 \mathrm{D}$ Jordan totient matrix $\mathfrak{F}^{t}$ as follows:

$$
\begin{aligned}
& \mathfrak{J}_{\infty}^{t}=\left\{x=\left(x_{m n}\right) \in \Omega: \sup _{m, n \in \mathbb{N}}\left|\left(\mathfrak{J}^{t} x\right)_{m n}\right|<\infty\right\}, \\
& \mathfrak{J}_{p}^{t}=\left\{x=\left(x_{m n}\right) \in \Omega: \exists \kappa \in \mathbb{C} \ni p-\lim _{m, n \rightarrow \infty}\left|\left(\mathfrak{J}^{t} x\right)_{m n}-\kappa\right|=0\right\}, \\
& \widetilde{\Im}_{b p}^{t}=\left\{x=\left(x_{m n}\right) \in \Omega: \mathfrak{\Im}^{t} x \in \mathscr{C}_{b p}\right\}, \\
& \mathfrak{\Im}_{r}^{t}=\left\{x=\left(x_{m n}\right) \in \Omega: \mathfrak{\Im}^{t} x \in \mathscr{C}_{r}\right\}, \\
& \mathfrak{J}_{s}^{t}=\left\{x=\left(x_{m n}\right) \in \Omega: \sum_{m, n}\left|\left(\mathfrak{J}^{t} x\right)_{m n}\right|^{s}<\infty\right\} .(0<s<\infty) .
\end{aligned}
$$

The sets which are newly defined above can be rewritten as $\mathfrak{\Im}_{\infty}^{t}=\left(\mathscr{M}_{u}\right)_{\mathfrak{J}^{t}}, \mathfrak{\Im}_{\vartheta}^{t}=\left(\mathscr{C}_{\vartheta}\right)_{\mathfrak{J}^{t}}$, and $\mathfrak{\Im}_{s}^{t}=\left(\mathscr{L}_{s}\right)_{\mathfrak{J}^{t}}$ with the notation (8), where $\vartheta \in\{p, b p, r\}$.

Theorem 4. The sets $\mathfrak{\Im}_{\infty}^{t}, \mathfrak{\Im}_{p}^{t}, \mathfrak{\Im}_{b p}^{t}$, $\mathfrak{J}_{r}^{t}$, and $\mathfrak{\Im}_{s}^{t}$ are linear spaces, and the following statements hold:

(i) The spaces $\mathfrak{\mho}_{\infty}^{t}, \mathfrak{\mho}_{b p}^{t}$, and $\mathfrak{\Im}_{r}^{t}$ are Banach spaces with

$$
\|x\|_{\mathfrak{J}_{\infty}^{t}}=\left\|\mathfrak{J}^{t} x\right\|_{\infty}=\sup _{m, n \in \mathbb{N}}\left|\frac{1}{(m n)^{t}} \sum_{k|m, l| n} J_{t}(k) J_{t}(l) x_{k l}\right|,
$$

which are linearly norm isomorphic to $\mathscr{M}_{u}, \mathscr{C}_{b p}$, and $\mathscr{C}_{r}$, respectively.

(ii) The space $\mathfrak{\Im}_{p}^{t}$ is a complete seminormed space with

$$
\|x\|_{\mathfrak{J}_{p}^{t}}=\lim _{i \rightarrow \infty}\left(\sup _{m, n \geq i}\left|\left(\mathfrak{J}^{t} x\right)_{m n}\right|\right),
$$

which is linearly isomorphic to the space $\mathscr{C}_{p}$.

(iii) For $0<s<1, \mathfrak{\Im}_{s}^{t}$ is a complete s-normed space with

$$
\|x\|_{\mathfrak{J}_{s}^{t}}^{2}=\left\|\mathfrak{\Im}^{t} x\right\|_{\mathscr{L}_{s}}^{2}=\sum_{m, n}\left|\frac{1}{(m n)^{t}} \sum_{k|m, l| n} J_{t}(k) J_{t}(l) x_{k l}\right|^{s},
$$

(iv) For $1 \leq s<\infty, \mathfrak{\Im}_{s}^{t}$ is a Banach space with

$$
\|x\|_{\mathfrak{J}_{s}^{t}}=\left\|\mathfrak{J}^{t} x\right\|_{s}=\left(\sum_{m, n}\left|\frac{1}{(m n)^{t}} \sum_{k|m, l| n} J_{t}(k) J_{t}(l) x_{k l}\right|^{s}\right)^{1 / s},
$$

which is linearly norm isomorphic to the space $\mathscr{L}_{s}$.

Proof. Since the other items of the theorem can be proven with similar technique, we will pass the others except first one for the set $\mathfrak{\Im}_{\infty}^{t}$.

It is left to the reader to show that $\mathfrak{\Im}_{\infty}^{t}$ is a normed linear space. To confirm the fact that $\mathfrak{\Im}_{\infty}^{t}$ is linearly norm isomorphic to $\mathscr{M}_{u}$, it is needed to sure the presence of any linear and norm keeping bijection from $\mathfrak{\Im}_{\infty}^{t}$ to $\mathscr{M}_{u}$. Let us describe the transformation $L: \mathfrak{J}_{\infty}^{t} \longrightarrow \mathscr{M}_{u}$ such that $L(x)=\mathfrak{J}^{t} x$ for all $x \in \mathfrak{J}_{\infty}^{t}$. It is easy to see the linearity of $L$. Furthermore, we can understand the injectivity of $L$ from the fact that $L x=$ $0 \Rightarrow x=0$. Let us suppose the sequences $y=\left(y_{k l}\right) \in \mathscr{M}_{u}$ and $x=\left(x_{m n}\right)$ whose terms are

$$
x_{m n}=\sum_{k|m, l| n} \frac{\mu(m / k) \mu(n / l)}{J_{t}(m) J_{t}(n)}(k l)^{t} y_{k l} \text {. }
$$

So, we get

$$
\begin{aligned}
\left(\mathfrak{J}^{t} x\right)_{m n} & =\frac{1}{(m n)^{t}} \sum_{k|m, l| n} J_{t}(k) J_{t}(l) x_{k l} \\
& =\frac{1}{(m n)^{t}} \sum_{k|m, l| n} J_{t}(k) J_{t}(l) \sum_{i|k, r| l} \frac{\mu(k / i) \mu(l / r)}{J_{t}(k) J_{t}(l)}(i r)^{t} y_{i r} \\
& =\frac{1}{(m n)^{t}} \sum_{k|m, l| n i|k, r| l} \sum_{(k / i) \mu(l / r)(i r)^{t} y_{i r}=y_{m n}}
\end{aligned}
$$

which yields us that $\sup _{m, n}\left|\left(\mathfrak{\Im}^{t} x\right)_{m n}\right|=\sup _{m, n}\left|y_{m n}\right|$. Thus, it is obtained that $x \in \mathfrak{\Im}_{\infty}^{t}$, since $y \in \mathscr{M}_{u}$. That is, $L$ is surjective and since the equality $\|x\|_{\mathfrak{J}_{\infty}^{t}}=\left\|\mathfrak{J}^{t} x\right\|_{\infty}$ holds, $L$ keeps the norm. Moreover, $\mathscr{M}_{u}$ is a Banach space, and we obtain the desired result from Section (b) of Corollary 6.3.41 in [34].

Now, we may give two theorems and a lemma related the inclusion relations.

Theorem 5. $\mathscr{M}_{u} \subset \mathfrak{\Im}_{\infty}^{t}$.

Proof. Consider $x \in \mathscr{M}_{u}$. Since,

$$
\begin{aligned}
\|x\|_{\mathfrak{J}_{\infty}^{t}} & =\sup _{m, n \in \mathbb{N}}\left|\frac{1}{(m n)^{t}} \sum_{k|m, l| n} J_{t}(k) J_{t}(l) x_{k l}\right| \\
& \leq\|x\|_{\infty} \sup _{m, n \in \mathbb{N}}\left|\frac{1}{(m n)^{t}} \sum_{k|m, l| n} J_{t}(k) J_{t}(l)\right|=\|x\|_{\infty}<\infty,
\end{aligned}
$$

which is linearly s-norm isomorphic to the space $\mathscr{L}_{s}$. it is obtained that $x \in \mathfrak{\Im}_{\infty}^{t}$, and thus, $\mathscr{M}_{u}$ is subspace of $\mathfrak{\Im}_{\infty}^{t}$. 
TABLE 2: Spaces and their duals which are computed in this section.

\begin{tabular}{lccccc}
\hline $\mathfrak{\Im}_{\infty}^{t}$ & $\mathfrak{J}_{p}^{t}$ & $\mathfrak{J}_{b p}^{t}$ & $\mathfrak{J}_{r}^{t}$ & $\mathfrak{J}_{s}^{t}(0<s \leq 1)$ & $\mathfrak{J}_{s}^{t}(1<s<\infty)$ \\
\hline$\alpha, \beta(p), \beta(b p), \gamma$ & $\beta(\vartheta)$ & $\alpha, \beta(\vartheta), \gamma$ & $\beta(\vartheta)$ & $\alpha, \beta(b p), \gamma$ & $\beta(b p), \gamma$ \\
\hline
\end{tabular}

Now, we give a lemma which is needed to prove our results.

Lemma 6 (see [35]). Suppose that $B=\left(b_{\text {mnkl }}\right)$ is a $4 D$ infinite matrix. Then, $B \in\left(\mathscr{C}_{p}: \mathscr{C}_{\vartheta}\right)$ if and only if following conditions hold:

$$
\begin{gathered}
\sup _{m, n \in \mathbb{N}} \sum_{k, l}\left|b_{m n k l}\right|<\infty, \\
\exists a_{k l} \in \mathbb{C} \ni \vartheta-\lim _{m, n \longrightarrow \infty} b_{m n k l}=a_{k l} \text { subsists, } \\
\exists \kappa \in \mathbb{C} \ni \vartheta-\lim _{m, n \longrightarrow \infty} \sum_{k, l} b_{m n k l}=\kappa \text { subsists, } \\
\forall k \in \mathbb{N}, \exists l_{0} \ni b_{m n k l}=0, \quad \forall l>l_{0}, \\
\forall l \in \mathbb{N}, \exists k_{0} \ni b_{m n k l}=0, \quad \forall k>k_{0} .
\end{gathered}
$$

Theorem 7. $\mathscr{C}_{p}$ is the subspace of $\mathfrak{\Im}_{\vartheta}^{t}$, where $\vartheta \in\{p, b p, r\}$.

Proof. To show the validity of the theorem, we just need to show that the 4D Jordan totient matrix $\mathfrak{J}^{t}$ satisfies the Lemma 6's conditions.

From the equality $\sup _{m, n \in \mathbb{N}} \sum_{k, l}\left|j_{m n k l}^{t}\right|=\sup _{m, n \in \mathbb{N}} \sum_{k, l} \mid J_{t}$ $(k) J_{t}(l) /(m n)^{t} \mid=1$, (24) holds. Since $\mathfrak{F}^{t}$ is RH-regular, then (25) holds. From the equality $\vartheta-\lim _{m, n \rightarrow \infty} \sum_{k, l} j_{m n k l}^{t}=1$, (26) holds. For all $k \in \mathbb{N}$, if we take $l_{0}=n$, then $j_{m n k l}^{t}=0$ for all $l>l_{0}$ and $m, n \in \mathbb{N}$. Therefore, (27) holds. The condition (28) can be shown similarly with (27).

\section{Duals}

Duals of a $\Psi \subset \Omega$ are described by the following way:

$$
\begin{aligned}
\Psi^{\alpha}: & =\left\{c=\left(c_{k l}\right) \in \Omega: \sum_{k, l}\left|c_{k l} x_{k l}\right|<\infty \text { for all }\left(x_{k l}\right) \in \Psi\right\}, \\
\Psi^{\beta(9)}: & =\left\{c=\left(c_{k l}\right) \in \Omega: \vartheta-\sum_{k, l} c_{k l} x_{k l} \text { subsists for all }\left(x_{k l}\right) \in \Psi\right\}, \\
\Psi^{\gamma}: & =\left\{c=\left(c_{k l}\right) \in \Omega: \sup _{m, n \in \mathbb{N}}\left|\sum_{k, l=1}^{m, n} c_{k l} x_{k l}\right|<\infty \text { for all }\left(x_{k l}\right) \in \Psi\right\} .
\end{aligned}
$$

Note that $\Psi^{\alpha} \subset \Psi^{\gamma}$ and if $\Psi \subset \Lambda$, then $\Lambda^{\alpha} \subset \Psi^{\alpha}$.

It is listed the spaces and their duals which are computed in this section in Table 2.
Theorem 8. The following statements hold:

$$
\begin{aligned}
& \left(\mathfrak{\Im}_{\infty}^{t}\right)^{\alpha}=\mathscr{L}_{u}, \\
& \left(\mathfrak{J}_{b p}^{t}\right)^{\alpha}=\mathscr{L}_{u} .
\end{aligned}
$$

Proof.

(i) Since it can be proven by applying a similar way used in Theorem 3.1 in [7], we omit details

(ii) For this part of the proof, it will be used contradiction method. Let us take a sequence $c \in\left(\mathfrak{J}_{b p}^{t}\right)^{\alpha} \backslash \mathscr{L}_{u}$. In that case, $\sum_{m, n}\left|c_{m n} x_{m n}\right|<\infty$ for all $x \in \mathfrak{\Im}_{b p}^{t}$. Consider that $e=\sum_{m, n} e^{m n} \in \mathfrak{J}_{b p}^{t}$. Thus, we obtain that $c e=$ $c \notin \mathscr{L}_{u}$, that is $c \notin\left(\mathfrak{\Im}_{b p}^{t}\right)^{\alpha}$. Therefore, it must be $c \epsilon$ $\mathscr{L}_{u}$ and consequently the inclusion $\left(\mathfrak{J}_{b p}^{t}\right)^{\alpha} \subset \mathscr{L}_{u}$ holds

Conversely, let us take $c=\left(c_{m n}\right) \in \mathscr{L}_{u}$ and $x \in \mathfrak{\Im}_{b p}^{t}$. In this fact, we understand that $y \in \mathscr{C}_{b p}$ from the equality (10), and therefore, $\sup _{m n}\left|y_{m n}\right| \leq K$. Thus, we obtain from the following inequality

$$
\begin{aligned}
\sum_{m, n}\left|c_{m n} x_{m n}\right| & =\sum_{m, n}\left|c_{m n}\right|\left|\sum_{k|m, l| n} \frac{\mu(m / k) \mu(n / l)}{J_{t}(m) J_{t}(n)}(k l)^{t} y_{k l}\right| \\
& \leq K \sum_{m, n}\left|c_{m n}\right|
\end{aligned}
$$

that $c \in\left(\mathfrak{\Im}_{b p}^{t}\right)^{\alpha}$ and this completes the proof.

Let us state the following conditions which will be used to give the $4 \mathrm{D}$ martix classes (see $[2,8,10,22,32,35-37]$ ).

$$
\begin{gathered}
\sup _{m, n, k, l \in \mathbb{N}}\left|b_{m n k l}\right|<\infty, \\
\sup _{m, n \in \mathbb{N}} \sum_{k, l}\left|b_{m n k l}\right|^{s^{\prime}}<\infty, \\
\sup _{k, l \in \mathbb{N}} \sum_{m, n}\left|b_{m n k l}\right|^{s_{1}}<\infty, \\
\exists k_{0} \in \mathbb{N} \ni \vartheta-\lim _{m, n \rightarrow \infty} \sum_{l}\left|b_{m n, k_{0}, l}-a_{k_{0}}\right|=0, \\
\exists l_{0} \in \mathbb{N} \ni \vartheta-\lim _{m, n \rightarrow \infty} \sum_{k}\left|b_{m n k, l_{0}}-a_{k, l_{0}}\right|=0, \\
\exists l_{0} \in \mathbb{N} \ni \vartheta-\lim _{m, n \rightarrow \infty} \sum_{k} b_{m n k, l_{0}}=\rho_{l_{0}},
\end{gathered}
$$


TABLe 3: Characterizations of $(\Psi: \Lambda)$, where $\Psi, \Lambda \in\left\{\mathscr{M}_{u}, \mathscr{C}_{p}, \mathscr{C}_{b p}, \mathscr{C}_{r}, \mathscr{L}_{s}\right\}$.

\begin{tabular}{lcccc}
\hline$(\Psi \downarrow: \Lambda \rightarrow)$ & $\mathscr{M}_{u}$ & $\mathscr{C}_{p}$ & $\mathscr{C}_{b p}$ & $\mathscr{C}_{r}$ \\
\hline $\mathscr{M}_{u}$ & $(5)$ & $(6),(8),(9)$ & $(5),(6),(17),(18),(19),(20)$ & $\bullet$ \\
$\mathscr{C}_{p}$ & $\bullet$ & $(5),(6),(7),(8),(9)$ & $(5),(6),(7),(8),(9)$ & $(5),(6),(7),(8),(9)$ \\
$\mathscr{C}_{b p}$ & $(5)$ & $(5),(6),(7),(13),(14)$ & $(5),(6),(7),(13),(14)$ & $(5),(6),(7),(13),(14)$ \\
$\mathscr{C}_{r}$ & $\bullet$ & $(5),(6),(7),(15),(16)$ & $(5),(6),(7),(15),(16)$ & $(5),(6),(7),(15),(16)$ \\
$\mathscr{L}_{s}(0<s \leq 1)$ & $(10)$ & $\bullet$ & $(6),(10)$ & $\bullet$ \\
$\mathscr{L}_{s}(1<s<\infty)$ & $(11)$ & $\bullet$ & $(6),(11)$ & $\bullet$ \\
\hline
\end{tabular}

The symbol $\bullet$ represents unknown conditions for $(\Psi: \Lambda)$.

$$
\begin{array}{r}
\exists k_{0} \in \mathbb{N} \ni \vartheta-\lim _{m, n \rightarrow \infty} \sum_{l} b_{m n, k_{0}, l}=\rho_{k_{0}}, \\
\exists a_{k l} \in \mathbb{C} \ni b p-\lim _{m, n \rightarrow \infty} \sum_{k, l}\left|b_{m n k l}-a_{k l}\right|=0, \\
b p-\lim _{m, n \rightarrow \infty} \sum_{k=1}^{m} b_{m n k l} \text { subsists, } \quad \forall l \in \mathbb{N}, \\
b p-\lim _{m, n \rightarrow \infty} \sum_{l=1}^{n} b_{m n k l} \text { subsists, } \quad \forall k \in \mathbb{N}, \\
\sum_{k, l}\left|b_{m n k l}\right| \text { converges. }
\end{array}
$$

It is listed necessary and sufficient conditions of $(\Psi: \Lambda)$ with the following table.

Theorem 9. Consider the set F defined by

$$
F=\left\{c=\left(c_{m n}\right) \in \Omega: \sup _{k, l \in \mathbb{N}} \sum_{m, n}\left|\frac{\mu(m / k) \mu(n / l)}{J_{t}(m) J_{t}(n)}(k l)^{t} c_{m n}\right|<\infty\right\} .
$$

$$
\text { If } 0<s \leq 1 \text {, then }\left(\widetilde{\Im}_{s}^{t}\right)^{\alpha}=F \text {. }
$$

Proof. Let us describe the $4 \mathrm{D}$ matrix $G=\left(g_{m n k l}\right)$ by

$$
g_{m n k l}:= \begin{cases}\frac{\mu(m / k) \mu(n / l)}{J_{t}(m) J_{t}(n)}(k l)^{t} c_{m n}, & k|m, l| n, \\ 0, & \text { for the other cases. }\end{cases}
$$

In this fact, by using the equality (12), we obtain that

$$
\begin{aligned}
c_{m n} x_{m n} & =c_{m n} \sum_{k|m, l| n} \frac{\mu(m / k) \mu(n / l)}{J_{t}(m) J_{t}(n)}(k l)^{t} y_{k l} \\
& =\sum_{k|m, l| n}\left(\frac{\mu(m / k) \mu(n / l)}{J_{t}(m) J_{t}(n)}(k l)^{t} c_{m n}\right) y_{k l}=(G y)_{m n} .
\end{aligned}
$$

Thus, we conclude from the relation (35) that $c x \in \mathscr{L}_{u}$ whenever $x \in \mathfrak{\Im}_{s}^{t}$ if and only if $G y \in \mathscr{L}_{u}$ whenever $y \in \mathscr{L}_{s}$. This fact implies that $c \in\left(\mathfrak{\Im}_{s}^{t}\right)^{\alpha}$ if and only if $G \in\left(\mathscr{L}_{s}: \mathscr{L}_{u}\right)$. Therefore, by using the condition of the class $\left(\mathscr{L}_{s}: \mathscr{L}_{s_{1}}\right)$ for $0<s \leq 1$ and $s_{1}=1$ in Table 3 with the matrix $G=\left(g_{m n k l}\right)$ instead of $B=\left(b_{m n k l}\right)$, we obtain the desired consequence.

Theorem 10. Let us take $\Psi \subset \Omega, c=\left(c_{m n}\right) \in \Omega$ and the $4 D$ matrix $O=\left(o_{\text {mnkl }}\right)$ as

$o_{m n k l}:= \begin{cases}\sum_{a=k, k \mid a}^{m} \sum_{b=l, l \mid b}^{n} \frac{\mu(a / k) \mu(b / l)}{J_{t}(a) J_{t}(b)}(k l)^{t} c_{a b}, & 1 \leq k \leq m, 1 \leq l \leq n, \\ 0, & \text { otherwise. }\end{cases}$

In that case,

$$
\begin{gathered}
\left(\Psi_{\mathfrak{J}^{t}}\right)^{\beta(\vartheta)}=\left\{c=\left(c_{m n}\right) \in \Omega: O \in\left(\Psi: \mathscr{C}_{\vartheta}\right)\right\} \\
\left(\Psi_{\mathfrak{J}^{t}}\right)^{\gamma}=\left\{c=\left(c_{m n}\right) \in \Omega: O \in\left(\Psi: \mathscr{M}_{u}\right)\right\}
\end{gathered}
$$

Proof.

(i) Let us take sequences $c=\left(c_{m n}\right) \in \Omega$ and $x \in \Psi_{\mathfrak{F}^{t}}$. In that case, $y=\left(y_{m n}\right) \in \Psi$ with $\mathfrak{\Im}^{t} x=y$. So, from the equality (12),

$$
\begin{aligned}
z_{m n} & =\sum_{k, l=1}^{m, n} c_{k l} x_{k l}=\sum_{k, l=1}^{m, n} c_{k l} \sum_{a|k, b| l} \frac{\mu(k / a) \mu(l / b)}{J_{t}(k) J_{t}(l)}(a b)^{t} y_{a b} \\
& =\sum_{k, l=1}^{m, n}\left[\sum_{a=k, k \mid a}^{m} \sum_{b=l, l \mid b}^{n} \frac{\mu(a / k) \mu(b / l)}{J_{t}(a) J_{t}(b)}(k l)^{t} c_{a b}\right] y_{k l}=(O y)_{m n},
\end{aligned}
$$

for all $m, n \in \mathbb{N}$. Then, by considering the equality above, we deduce that $c x \in \mathscr{C} \mathcal{S}_{\vartheta}$ whenever $x \in \Psi_{\mathfrak{J}^{t}}$ if and only if $z \in \mathscr{C}_{\vartheta}$ whenever $y \in \Psi$. This leads us to the fact that $c \in\left(\Psi_{\mathfrak{J}^{t}}\right)^{\beta(\vartheta)}$ if and only if $O \in\left(\Psi: \mathscr{C}_{\vartheta}\right)$, as claimed. 
(ii) This part can be easily proven similarly to the first part by using the description of the $\gamma$-dual. So, we omit the proof.

Now, consider the sets $\varpi_{f}$ which are defined by

$\omega_{f}=\left\{c=\left(c_{m n}\right) \in \Omega:\right.$ Condition $(f)$ holds with $o_{m n k l}$ instead of $\left.b_{m n k l}\right\}$,

where the $4 \mathrm{D}$ matrix $O=\left(o_{\text {mnkl }}\right)$ defined as in Theorem 10 and $5 \leq f \leq 20$.

Theorem 11. The following statements hold:

$$
\begin{aligned}
& \left(\mathfrak{\mho}_{p}^{t}\right)^{\beta(9)}=\bigcap_{f=5}^{9} \varpi_{f}, \\
& \left(\mathfrak{J}_{b p}^{t}\right)^{\beta(9)}=\bigcap_{f=5}^{7} \varpi_{f} \cap \varpi_{13} \cap \varpi_{14}, \\
& \left(\mathfrak{J}_{r}^{t}\right)^{\beta(9)}=\bigcap_{f=5}^{7} \varpi_{f} \cap \varpi_{15} \cap \varpi_{16} .
\end{aligned}
$$

Proof. From Theorem 10 and the conditions of the classes $\left(\mathscr{C}_{p}: \mathscr{C}_{\vartheta}\right),\left(\mathscr{C}_{b p}: \mathscr{C}_{\vartheta}\right)$, and $\left(\mathscr{C}_{r}: \mathscr{C}_{\vartheta}\right)$ in Table 3 with $o_{m n k l}$ instead of $b_{m n k l}$, the accuracy of items (i), (ii), and (iii) can be easily seen, respectively.

Theorem 12. The following statements hold:

$$
\left(\widetilde{\Im}_{\infty}^{t}\right)^{\beta(b p)}=\omega_{5} \cap \omega_{6} \bigcap_{f=17}^{20} \omega_{f} .
$$

(i) For $0<s \leq 1,\left(\mathfrak{\Im}_{s}^{t}\right)^{\beta(b p)}=\varpi_{6} \cap \varpi_{10}$

(ii) For $1<s<\infty,\left(\widetilde{\mho}_{s}^{t}\right)^{\beta(b p)}=\omega_{6} \cap \varpi_{11}$

$$
\left(\mathfrak{\Im}_{\infty}^{t}\right)^{\beta(p)}=\varpi_{6} \cap \varpi_{8} \cap \varpi_{9}
$$

Proof. From Theorem 10 and the conditions of the classes $\left(\mathscr{M}_{u}: \mathscr{C}_{b p}\right),\left(\mathscr{L}_{s}: \mathscr{C}_{b p}\right)$ for $0<s \leq 1,\left(\mathscr{L}_{s}: \mathscr{C}_{b p}\right)$ for $1<s<$ $\infty$ and $\left(\mathscr{M}_{u}: \mathscr{C}_{p}\right)$ in Table 3 with $o_{\text {mnkl }}$ instead of $b_{\text {mnkl }}$, the accuracy of items (i), (ii), (iii), and (iv) can be easily seen, respectively.
Theorem 13. The following statements hold:

$$
\begin{gathered}
\left(\mathfrak{\mho}_{\infty}^{t}\right)^{\gamma}={\varpi_{5},} \\
\left(\mathfrak{\Im}_{b p}^{t}\right)^{\gamma}=\varpi_{5} .
\end{gathered}
$$

(i) For $0<s \leq 1,\left(\mathfrak{\Im}_{s}^{t}\right)^{\gamma}=\varpi_{10}$

(ii) For $1<s<\infty,\left(\mathfrak{\mho}_{s}^{t}\right)^{\gamma}=\varpi_{11}$

Proof. From Theorem 10 and the conditions of the classes $\left(\mathscr{M}_{u}: \mathscr{M}_{u}\right),\left(\mathscr{C}_{b p}: \mathscr{M}_{u}\right),\left(\mathscr{L}_{s}: \mathscr{M}_{u}\right)$ for $0<s \leq 1$, and $\left(\mathscr{L}_{s}:\right.$ $\mathscr{M}_{u}$ ) for $1<s<\infty$ in Table 3 with $o_{m n k l}$ instead of $b_{m n k l}$, the accuracy of items (i), (ii), (iii), and (iv) can be easily seen, respectively.

\section{4D Matrix Transformations}

In the current part of the article, it will be given the classes $\left(\Psi_{\mathfrak{J}^{t}}: \Lambda\right)$ and $\left(\Psi: \Lambda_{\mathfrak{J}^{t}}\right)$, where $\Psi, \Lambda \in\left\{\mathscr{M}_{u}, \mathscr{C}_{b p}, \mathscr{C}_{p}, \mathscr{C}_{r}\right.$, $\left.\mathscr{L}_{s}\right\}$.

Theorem 14. Consider the $4 D$ infinite matrices $B=\left(b_{\text {mnkl }}\right)$ and $H=\left(h_{m n k l}\right)$ whose elements are connected with the equality

$$
h_{\text {mnkl }}=\sum_{a=k, k \mid a}^{\infty} \sum_{b=l, l \mid b}^{\infty} \frac{\mu(a / k) \mu(b / l)}{J_{t}(a) J_{t}(b)}(k l)^{t} b_{m n a b} .
$$

In that case; $B \in\left(\Psi_{\widetilde{J}^{t}}: \Lambda\right)$ if and only if $B_{m n} \in\left(\Psi_{\mathfrak{J}^{t}}\right)^{\beta(9)}$ and $H \in(\Psi: \Lambda)$, where $\Psi, \Lambda \in\left\{\mathscr{M}_{u}, \mathscr{C}_{b p}, \mathscr{C}_{p}, \mathscr{C}_{r}, \mathscr{L}_{s}\right\}$.

Proof. Assume that $B \in\left(\Psi_{\mathfrak{J}^{t}}: \Lambda\right)$. In that case, $B x$ exists and is in $\Lambda$ for all $x \in \Psi_{\widetilde{J}^{t}}$ such that $y=\mathfrak{J}^{t} x \in \Psi$, and it also implies that $B_{m n} \in\left(\Psi_{\mathfrak{S}^{t}}\right)^{\beta(9)}$. By bearing in mind (12), we obtain that

$$
\begin{aligned}
\sum_{k, l=1}^{i, j} b_{m n k l} x_{k l} & =\sum_{k, l=1}^{i, j} b_{m n k l}\left[\sum_{a|k, b| l} \frac{\mu(k / a) \mu(l / b)}{J_{t}(k) J_{t}(l)}(a b)^{t} y_{a b}\right] \\
& =\sum_{k, l=1}^{i, j}\left[\sum_{a=k, k \mid a}^{i} \sum_{b=l, l l \mid b}^{j} \frac{\mu(a / k) \mu(b / l)}{J_{t}(a) J_{t}(b)}(k l)^{t} b_{m n a b}\right] y_{k l},
\end{aligned}
$$

for all $i, j \in \mathbb{N}$. In that case, if we take 9 -limit on the equality (45) as $i, j \longrightarrow \infty$, we have $B x=H y$. Therefore, we obtain that $H y \in \Lambda$ whenever $y \in \Psi$, that is $H \in(\Psi: \Lambda)$.

Conversely, suppose that $B_{m n} \in\left(\Psi_{\widetilde{J}^{t}}\right)^{\beta(9)}$ and $H \in(\Psi$ : $\Lambda$ ). Let us take the sequence $x \in \Psi \mathfrak{J}^{t}$ with $y=\mathfrak{J}^{t} x \in \Psi$. In that 
TABLe 4: Characterizations of $\left(\Psi_{\mathfrak{J}^{t}}: \Lambda\right)$, where $\Psi, \Lambda \in\left\{\mathscr{M}_{u}, \mathscr{C}_{b p}, \mathscr{C}_{p}, \mathscr{C}_{r}, \mathscr{L}_{s}\right\}$.

\begin{tabular}{lcccc}
\hline$\left(\Psi_{\left.\mathfrak{J}^{t} \downarrow: \Lambda \rightarrow\right)}\right.$ & $\mathscr{M}_{u}$ & $\mathscr{C}_{p}$ & $\mathscr{C}_{b p}$ & $\mathscr{C}_{r}$ \\
\hline $\mathfrak{J}_{\infty}^{t}$ & $(5)$ & $(6),(8),(9)$ & $(5),(6),(17),(18),(19),(20)$ & $\bullet$ \\
$\mathfrak{J}_{p}^{t}$ & $\bullet$ & $(5),(6),(7),(8),(9)$ & $(5),(6),(7),(8),(9)$ & $(5),(6),(7),(8),(9)$ \\
$\mathfrak{J}_{b p}^{t}$ & $(5)$ & $(5),(6),(7),(13),(14)$ & $(5),(6),(7),(13),(14)$ & $(5),(6),(7),(13),(14)$ \\
$\mathfrak{J}_{r}^{t}$ & $\bullet$ & $(5),(6),(7),(15),(16)$ & $(5),(6),(7),(15),(16)$ & $(5),(6),(7),(15),(16)$ \\
$\mathfrak{J}_{s}^{t}(0<s \leq 1)$ & $(10)$ & $\bullet$ & $(6),(10)$ & $\bullet$ \\
$\mathfrak{J}_{s}^{t}(1<s<\infty)$ & $(11)$ & $\bullet$ & $(6),(11)$ & $\bullet$ \\
\hline
\end{tabular}

Holds with $h_{m n k l}$ instead of $b_{m n k l}$.

TABLe 5: Characterizations of $\left(\Psi: \Lambda_{\widetilde{J}^{t}}\right)$, where $\Psi, \Lambda \in\left\{\mathscr{M}_{u}, \mathscr{C}_{b p}, \mathscr{C}_{p}, \mathscr{C}_{r}, \mathscr{L}_{s}\right\}$.

\begin{tabular}{lcccc}
\hline$\left(\Psi \downarrow: \Lambda_{\left.\mathfrak{J}^{t} \rightarrow\right)}\right.$ & $\mathfrak{J}_{\infty}^{t}$ & $\mathfrak{J}_{p}^{t}$ & $\mathfrak{J}_{b p}^{t}$ & $\mathfrak{J}_{r}^{t}$ \\
\hline $\mathscr{M}_{u}$ & $(5)$ & $(6),(8),(9)$ & $(5),(6),(17),(18),(19),(20)$ & $\bullet$ \\
$\mathscr{C}_{p}$ & $\bullet$ & $(5),(6),(7),(8),(9)$ & $(5),(6),(7),(8),(9)$ & $(5),(6),(7),(8),(9)$ \\
$\mathscr{C}_{b p}$ & $(5)$ & $(5),(6),(7),(13),(14)$ & $(5),(6),(7),(13),(14)$ & $(5),(6),(7),(13),(14)$ \\
$\mathscr{C}_{r}$ & $\bullet$ & $(5),(6),(7),(15),(16)$ & $(5),(6),(7),(15),(16)$ & $(5),(6),(7),(15),(16)$ \\
$\mathscr{L}_{s}(0<s \leq 1)$ & $(10)$ & $\bullet$ & $(6),(10)$ & $\bullet$ \\
$\mathscr{L}_{s}(1<s<\infty)$ & $(11)$ & $\bullet$ & $(6),(11)$ & $\bullet$ \\
\hline
\end{tabular}

Holds with $u_{m n k l}$ instead of $b_{m n k l}$.

case, $B x$ exists. By using the relation (12), one can derive from the relation (45) when passing $\vartheta$-limit as $i, j \longrightarrow \infty$ that

$$
\sum_{k, l} b_{m n k l} x_{k l}=\sum_{k, l} h_{m n k l} y_{k l}
$$

which implies that $B x=H y$. Therefore, $B \in\left(\Psi_{\mathfrak{J}^{t}}: \Lambda\right)$, and this completes the proof.

Corollary 15. Consider the $4 D$ infinite matrices $B=\left(b_{m n k l}\right)$ and $H=\left(h_{m n k l}\right)$ whose elements are connected with the relation (44). In that case, the necessary and sufficient conditions for the classes $\left(\Psi_{\mathfrak{I}^{t}}: \Lambda\right)$ can be read from the Table 4.

Lemma 16 (see [8]). Let $\Psi, \Lambda \subset \Omega, B=\left(b_{\text {mnkl }}\right)$ be a $4 D$ matrix and $F=\left(f_{\text {mnkl }}\right)$ also be a $4 D$ triangle. In that case, $B \in$ $\left(\Psi: \Lambda_{F}\right)$ if and only if $F B \in(\Psi: \Lambda)$.

Now, let us define the $4 D$ matrix $U=\left(u_{\text {mnkl }}\right)$ by

$$
u_{m n k l}=\sum_{i|m, d| n} j_{m n i d}^{t} b_{i d k l},
$$

and give the following corollary.

Corollary 17. Consider the $4 D$ infinite matrices $B=\left(b_{\text {mnkl }}\right)$ and $U=\left(u_{m n k l}\right)$ whose elements are connected with the relation (47). In that case, the necessary and sufficient conditions for the classes $\left(\Psi: \Lambda_{\mathfrak{J}^{t}}\right)$ can be read from Table 5 .

\section{Conclusions}

As we have stated some of them in Table 1, double sequence spaces which are obtained by using the domains of triangular matrices have been studied many times by many authors in the past. In the light of these and similar studies, in the current study, we described a new $\mathrm{RH}$-regular and triangular $4 \mathrm{D}$ matrix derived by famous Jordan's function which is an arithmetic function and introduced some double sequence spaces by using this matrix on some classical double sequence spaces. Moreover, we investigated their some properties and inclusion relations related them, computed duals, and characterized some matrix classes. We conclude that the results obtained from the 4D Jordan totient matrix $\mathfrak{\Im}^{t}$ is more general and extensive than the existent results of the authors $[6,7]$. We expect that our results might be a reference for further studies in this field.

\section{Data Availability}

No data were used to support this study.

\section{Conflicts of Interest}

The authors declare that they have no conflicts of interest.

\section{References}

[1] L. E. Dickson, History of the Theory of Numbers, Chelsea Publishing Company, New York, 1971. 
[2] M. Zeltser, "On conservative matrix methods for double sequence spaces," Acta Mathematica Hungarica, vol. 95, no. 3, pp. 225-242, 2002.

[3] G. G. Lorentz, "A contribution to the theory of divergent sequences," Acta Mathematica, vol. 80, pp. 167-190, 1948.

[4] F. Mòricz and B. E. Rhoades, "Almost convergence of double sequences and strong regularity of summability matrices," Mathematical Proceedings of the Cambridge Philosophical Society, vol. 104, no. 2, pp. 283-294, 1988.

[5] M. İlkhan, N. Şimşek, and E. E. Kara, "A new regular infinite matrix defined by Jordan totient function and its matrix domain in $\ell_{p}$," Mathematical Methods in the Applied Sciences, pp. 1-12, 2020.

[6] S. Demiriz and S. Erdem, "Domain of Euler-totient matrix operator in the space $\mathcal{L}_{p}$," The Korean Journal of Mathematics, vol. 28 , no. 2, pp. 361-378, 2020.

[7] S. Erdem and S. Demiriz, "4-Dimensional Euler-totient matrix operator and some double sequence spaces," Mathematical Sciences and Applications E-Notes, vol. 8, no. 2, pp. 110-122, 2020.

[8] M. Yeşilkyagil and F. Başar, "Domain of Riesz Mean in the Space $\mathcal{L}_{p}$," Filomat, vol. 31, no. 4, pp. 925-940, 2017.

[9] G. Talebi, "Operator norms of four-dimensional Hausdorff matrices on the double Euler sequence spaces," Linear and Multilinear Algebra, vol. 65, no. 11, pp. 2257-2267, 2017.

[10] O. Tuğ, "Four-dimensional generalized difference matrix and some double sequence spaces," Journal of Inequalities and Applications, vol. 2017, no. 1, 2017.

[11] O. Tuğ, "On almost $B$-summable double sequence spaces," Journal of Inequalities and Applications, vol. 2018, no. 1, p. 19, 2018.

[12] O. Tuğ, V. Rakočević, and E. Malkowsky, "On the domain of the four-dimensional sequential band matrix in some double sequence spaces," Mathematics, vol. 8, no. 5, p. 789, 2020.

[13] S. Demiriz and S. Erdem, "Domain of binomial matrix in some spaces of double sequences," Punjab University Journal of Mathematics, vol. 52, no. 11, pp. 65-79, 2020.

[14] S. Erdem and S. Demiriz, "Almost convergence and 4dimensional binomial matrix," Konuralp Journal of Mathematics, vol. 8, no. 2, pp. 329-336, 2020.

[15] M. Yeşilkyagil and F. Başar, "AK( 9$)$-property of the spaces of double series," Bulletin of the Malaysian Mathematical Sciences Society, vol. 44, no. 2, pp. 881-889, 2021.

[16] M. Yeşilkyagil and F. Başar, "On the paranormed space of bounded variation double sequences," Bulletin of the Malaysian Mathematical Sciences Society, vol. 43, no. 3, pp. 27012712, 2020.

[17] M. Yeşilkyagil and F. Başar, "Domain of Euler mean in the space of absolutely $p$-summable double sequences with $0<p$ $<\infty$," Analysis in Theory and Applications, vol. 34, no. 3, pp. 241-252, 2018.

[18] M. Yeşilkyagil and F. Başar, "Domain of Riesz mean in some spaces of double sequences," Indagationes Mathematicae, vol. 29, no. 3, pp. 1009-1029, 2018.

[19] M. Yeşilkyagil and F. Başar, "A note on Abel summability of double series," Numerical Functional Analysis and Optimization, vol. 38, no. 8, pp. 1069-1076, 2017.

[20] M. Yeșilkyagil and F. Bașar, "A note on Riesz summability of double series," Proceedings of the National Academy of Sciences, India Section A: Physical Sciences, vol. 86, no. 3, pp. 333-337, 2016.
[21] M. Yeşilkyagil and F. Başar, "Some topological properties of the spaces of almost null and almost convergent double sequences," Turkish Journal of Mathematics, vol. 40, no. 3, pp. 624-630, 2016.

[22] M. Yeşilkyagil and F. Başar, "Mercerian theorem for four dimensional matrices," Communications, vol. 65, no. 1, pp. 147-155, 2016.

[23] M. Yeşilkyagil and F. Başar, "Comparison of four dimensional summability methods," The Aligarh Bulletin Of Mathematics, vol. 34, no. 1-2, pp. 1-11, 2015.

[24] M. Mursaleen and F. Başar, "Domain of Cesàro mean of order one in some spaces of double sequences," Studia Scientiarum Mathematicarum Hungarica, vol. 51, no. 3, pp. 335-356, 2014.

[25] C. R. Adams, "On non-factorable transformations of double sequences," Proceedings of the National Academy of Sciences, vol. 19 , no. 5, pp. 564-567, 1933.

[26] B. Altay and F. Başar, "Some new spaces of double sequences," Journal of Mathematical Analysis and Applications, vol. 309, no. 1, pp. 70-90, 2005.

[27] F. Basar and Y. Sever, "The space $\mathcal{L}_{q}$ of double sequences," Mathematical Journal of Okayama University, vol. 51, pp. 149-157, 2009.

[28] R. C. Cooke, Infinite Matrices and Sequence Spaces, Macmillan and Co, Limited, London, 1950.

[29] M. İlkhan and E. E. Kara, "A new Banach space defined by Euler totient matrix operator," Operators and Matrices, vol. 13, no. 2, pp. 527-544, 2019.

[30] F. Mòricz, "Extensions of the spaces $\mathrm{c}$ and $\mathrm{c}_{0}$ from single to double sequences," Acta Mathematica Hungarica, vol. 57, no. 1-2, pp. 129-136, 1991.

[31] M. Mursaleen and F. Başar, "Sequence spaces: topics in modern summability theory," in Series: Mathematics and its Applications, CRC Press, Taylor and Francis Group, Boca Raton, London New York, 2020.

[32] H. J. Hamilton, "Transformations of multiple sequences," Duke Mathematical Journal, vol. 2, pp. 29-60, 1936.

[33] G. M. Robison, "Divergent double sequences and series," Transactions of the American Mathematical Society, vol. 28, no. 1, pp. 50-73, 1926.

[34] J. Boos, Classical and Modern Methods in Summability, Oxford University Press, Newyork, 2000.

[35] M. Zeltser, M. Mursaleen, and S. A. Mohiuddine, "On almost conservative matrix mathods for double sequence spaces," Universitatis Debreceniensis, vol. 75, pp. 387-399, 2009.

[36] C. Çakan, B. Altay, and M. Mursaleen, "The $\sigma$-convergence and $\sigma$-core of double sequences," Applied Mathematics Letters, vol. 19, pp. 387-399, 2006.

[37] M. Yeşilkyagil and F. Başar, "On the characterization of a class of four dimensional matrices and Steinhaus type theorems," Kragujevac Journal of Mathematics, vol. 40, no. 1, pp. 35-45, 2016. 